\title{
An Integrated Electromechanical-Reversible Growth Heart Model for Simulating Cardiac Therapies
}

\author{
L.C. Lee • J. Sundnes · M. Genet • J.F. Wenk • S.T. Wall
}

Received: date / Revised version: date

\begin{abstract}
In recent years, a sizable number of cardiac growth and remodeling (G\&R) models have been proposed and were used to study the effects of heart diseases and treatments. So far, the majority of simulations using these models have been confined to a single cardiac phase, and do not take into account all the mechanical states that the heart undergoes in the entire cardiac cycle. These models also do not take into account the coupling between electrophysiology and mechanics. To address this issue, we describe the integration of a recently proposed reversible G\&R model to an existing strongly-coupled electromechanical model of the heart. A separation of timescale between growth deformation and elastic deformation was invoked in this integrated electromechanicalreversible growth heart model. To test our model, we applied the model to study the effects of myocardial infarction in a realistic left ventricular (LV) geometry using finite element method. We also simulate the effects of a novel therapy that is based on alteration of the infarct mechanical properties. We show that our proposed model is able to predict key features that are consistent with experiments. Specifically, we show that the presence of
\end{abstract}

\section{L.C. Lee}

Department of Mechanical Engineering, Michigan State University, East Lansing, MI, USA E-mail: lclee@egr.msu.edu

J. Sundnes, S.T. Wall

Simula Research Laboratory, Oslo, Norway E-mail: sundnes/samwall@simula.no

\section{Genet}

Institute of Biomedical Engineering ETH Zurich, Zurich, Switzerland E-mail: genet@biomed.ee.ethz.ch

J.F. Wenk

Department of Mechanical Engineering, University of Kentucky, Lexington, KY, USA E-mail: jonathan.wenk@uky.edu a non-contractile infarct leads to a dilation of the left ventricle that results in a rightward shift of the pressure volume loop. Our model also predicts that $\mathrm{G} \& \mathrm{R}$ is attenuated by a reduction in $\mathrm{LV}$ dilation when the infarct stiffness is increased.

Keywords Finite element modeling · Growth and remodeling - Cardiac electromechanics . Myocardial infarction · Bioinjection therapy.

\section{Introduction}

Heart disease is currently the leading cause of death in the world, and the total cost that is associated with this disease is expected to triple between the years 2010 and 2030 (Heidenreich et al. 2011). To combat the rising costs that are associated with heart diseases, a myriad of new treatments have been conceived over the recent decades. These treatments include the surgical ventricular restoration procedure (Athanasuleas et al. 2004), injection of biocompatible material to augment the left ventricular (LV) wall (Sabbah et al. 2009) and the implantation of a left ventricular partitioning device (LVAD) (Nikolic et al. 2009).

Although animal and clinical studies are often used to understand the mechanisms and effects of these novel treatments (and diseases), computational modeling is increasingly used to perform and supplement these studies (Wall et al. 2006; Kerckhoffs et al. 2008; Wenk et al. 2009; Klepach et al. 2012; Lee et al. 2013a,b; Genet et al. 2014; Hu et al. 2014; Lee et al. 2014a,b). The key advantage of using computational modeling is its versatility and low cost (Niederer and Smith 2012; Lee et al. 2014c). However, other than a few computational models (Klepach et al. 2012; Kerckhoffs et al. 2012a), most are only capable of sim- 
ulating the acute immediate response of a treatment; and are not capable of directly simulating the chronic long term response, i.e., the propensity of the treatment to reverse or attenuate pathological growth and remodeling (G\&R) of the heart. Because remodeling is one of the key determinants of the clinical course of heart failure (Cohn et al. 2000), while the reversal of this process (i.e., reverse remodeling) is often associated with a favorable prognosis in many diseases ( $\mathrm{Yu}$ et al. 2005; Hoshikawa et al. 2011; Williams et al. 2011), the ability to directly simulate the treatment effects on pathological cardiac G\&R can shed tremendous light on its efficacy. Specifically, the ability to do so can help determine whether a particular treatment can improve the patient's prognosis.

A number of cardiac G\&R constitutive models have been developed to date. The majority of these models were formulated based on the theoretical framework of volumetric growth (Rodriguez et al. 1994). Under this framework, changes in the heart geometry due to the collective local effects of cellular hypertrophy can be described mechanistically. Constitutive G\&R models constructed under this framework have been able to predict key features of the myocardium during eccentric and concentric hypertrophy (Göktepe et al. 2010a,b). Recently, a reversible cardiac G\&R constitutive model that is not only capable of describing cellular hypertrophy but also cellular atrophy was developed (Lee et al. 2015). This model is able to predict LV reverse remodeling when the mechanical loading is decreased, a key feature found in hearts implanted with LVAD. However, except in Kerckhoffs et al. (2012b), the simulation of G\&R using all these models have thus far been confined only to a single cardiac phase, usually end of diastole. As a result, these models are unable to take into account all the mechanical states that occur during the entire cardiac cycle.

In this work, we overcome this limitation by integrating the newly developed reversible G\&R constitutive model (Lee et al. 2015) to an existing strongly-coupled electromechanical model of the heart (Sundnes et al. 2014). We also demonstrate that the integrated electromechanical-reversible growth model can be applied to understand and predict the mechanisms/effects of heart disease treatments. The organization of this work is as follow. Following this section, the construction of an integrated electromechanical-reversible growth model is described in Section 2. Specifically, we showed that the construction of this model enables one take into account the bidirectional relationship between myocardial G\&R and the mechanics that occur during the entire cardiac cycle. In section 3 , we describe an application of the integrated model to simulate the chronic effects of a novel therapy that seeks to attenuate pathological LV remodeling by modifying the stiffness of a myocardial infarct (Morita et al. 2011; Fomovsky et al. 2011). The simulation results are then presented in the next section. In the final section, we discussed our results and showed that the simulation results are consistent with clinical observations and animal studies of myocardial infarction (MI) and the therapy itself.

\section{Methods}

\subsection{Reversible growth constitutive model}

The reversible growth constitutive model (Lee et al. 2015) was constructed under the volumetric growth framework in which the deformation gradient tensor $\boldsymbol{F}$ was multiplicatively decomposed into a growth tensor $\boldsymbol{F}_{g}$ and an elastic deformation tensor $\boldsymbol{F}_{e}$, i.e.

$\boldsymbol{F}=\boldsymbol{F}_{e} \cdot \boldsymbol{F}_{g}$.

Through this decomposition, local tissue growth and shape changes were described by the incompatible growth tensor $\boldsymbol{F}_{g}$. The elastic deformation tensor $\boldsymbol{F}_{e}$ restored the required compatibility of the deformation gradient tensor $\boldsymbol{F}$. To describe cellular hypertrophy occurring in the fiber direction $\boldsymbol{f}$ e.g., during eccentric hypertrophy (Gerdes et al. 1992), $\boldsymbol{F}_{g}$ was parameterized by a scalar growth multiplier $\theta$ via the following equation

$\boldsymbol{F}_{g}=(\theta-1) \boldsymbol{f} \otimes \boldsymbol{f}+\boldsymbol{I}$.

The following constitutive relationship was prescribed to describe the time $t$ evolution of the local growth multiplier $\theta$ :

$\frac{d \theta}{d t}=k\left(\theta, \lambda_{e}\right)\left(\lambda_{e}-\lambda_{h}\right)$

In the above equation, the driving stimulant for growth and reverse growth is the deviation of the elastic myofiber stretch (over one cardiac cycle) $\lambda_{e}=\sqrt{\boldsymbol{f} \cdot \boldsymbol{F}_{e}^{T} \boldsymbol{F}_{e} \cdot \boldsymbol{f}}$ from its corresponding homeostatic set point $\lambda_{h}$. For $k(\theta) \geq 0$ (the purpose of which is discussed below), local tissue growth $(\dot{\theta}>0)$ or shrinkage $(\dot{\theta}<0)$ occurs when the average elastic myofiber stretch $\lambda_{e}$ exceeds or falls below the homeostatic stretch $\lambda_{h}$, respectively.

The other function $k\left(\theta, \lambda_{e}\right)$ is a rate limiting function whose main purpose is to ensure that the growth multiplier $\theta$ lies within some prescribed 
limits of $\theta_{\min }$ and $\theta_{\max }$ (i.e, $\theta_{\min } \leq \theta \leq \theta_{\max }$ ). Its functional form is given as follows:

$$
k\left(\theta, \lambda_{e}\right)=\left\{\begin{array}{l}
\frac{1}{\tau_{g}}\left(\frac{\theta_{\max }-\theta}{\theta_{\max }-\theta_{\min }}\right)^{\gamma_{g}} \text { if }\left(\lambda_{e}-\lambda_{h}\right) \geq 0 \\
\frac{1}{\tau_{r g}}\left(\frac{\theta-\theta_{\min }}{\theta_{\max }-\theta_{\min }}\right)^{\gamma_{r g}} \text { if }\left(\lambda_{e}-\lambda_{h}\right)<0
\end{array}\right.
$$

The main construct of this function is that there are some physiological limits associated with cellular hypertrophy and atrophy. To ensure that $\theta$ lies within these limits, the above function has the features $k\left(\theta, \lambda_{e}\right) \rightarrow 0$ as $\theta \rightarrow \theta_{\text {min }}$ or $\theta \rightarrow \theta_{\text {max }}$. The parameters $\gamma_{g}$ and $\gamma_{r g}$ are the degrees of nonlinearity of tissue growth and shrinkage due to the deposition and removal of sarcomere units, respectively; whereas, $\tau_{g}$ and $\tau_{r g}$ are the timescales associated with tissue growth and shrinkage, respectively. We note that the reversible growth constitutive model described above was developed from previous models of cardiac G\&R (Göktepe et al. 2010a; Klepach et al. 2012). Those models, however, do not permit growth to be reversed.

\subsection{Electromechanical model}

The coupled cardiac electromechanics model that is capable of simulating the entire cardiac cycle has been previously described (Wall et al. 2012; Sundnes et al. 2014). In this model, cardiac electrophysiology was described using a standard bidomain model, cellular electromechanics was described by a fully coupled model (Winslow et al. 1999; Rice et al. 2008), whereas tissue mechanics was described by the balance of linear momentum equations with mechanical constitutive relations that are discussed next.

The mechanical behavior of the cardiac tissue was described using an active stress formulation In this formulation, the Cauchy stress tensor $\boldsymbol{\sigma}$ of the cardiac tissue is additively decomposed into a passive component $\boldsymbol{\sigma}_{p}$ and an active component $\boldsymbol{\sigma}_{a}$ that accounts for the crossbridge cycling process of the tissue upon excitation i.e.,

$\boldsymbol{\sigma}=\boldsymbol{\sigma}_{p}+\boldsymbol{\sigma}_{a}\left(\boldsymbol{s}, \lambda_{e}, \dot{\lambda}_{e} ; T\right)$.

The elastic deformation gradient tensor $\boldsymbol{F}_{e}$ in Eq. (1) enters both of these stress tensors $\boldsymbol{\sigma}_{p}$ and $\boldsymbol{\sigma}_{a}$. Specifically, the development of the active stress $\boldsymbol{\sigma}_{a}$ was described by the constitutive model proposed by Rice et al. (2008). In this model, cellular cross-bridge cycling and active tension development was described by a system of ordinary differential equations involving the state vector $\boldsymbol{s}$, elastic myofiber stretch $\lambda_{e}$, rate of elastic myofiber stretch $\dot{\lambda}_{e}$ and the reference tension $T$, a material parameter that scales the tissue contractility. The state vector $s$ serve as the coupling variable between electrophysiology and mechanics, and describe the states of various membrane channels and intracellular ionic concentration. Also, we note that this model was originally formulated at the sarcomere level without consideration of growth. Our choice of using the elastic myofiber stretch as variable to account for length dependence of the active force generated by the crossbridge formation is justified because growth (and reverse growth) of myocytes occurs via the addition (and removal) of sarcomere units and do not occur by lengthening (and shortening) of each individual sarcomere unit (Gerdes et al. 1992).

The passive stress $\boldsymbol{\sigma}_{p}$ was described using a Fung-type transversely isotropic hyperelastic constitutive model (Guccione et al. 1991) with the strain energy function

$W\left(\boldsymbol{E}_{e}\right)=\frac{C}{2}(\exp Q-1)+C_{\mathrm{compr}}\left(J^{e} \ln J^{e}-J^{e}+1\right)$,

where

$$
\begin{array}{r}
Q=b_{f} E_{f f}{ }^{2}+b_{s}\left(E_{s s}{ }^{2}+E_{n n}{ }^{2}+E_{s n}{ }^{2}+E_{n s}{ }^{2}\right) \\
+b_{f s}\left(E_{f s}{ }^{2}+E_{s f}{ }^{2}+E_{f n}{ }^{2}+E_{n f}{ }^{2}\right) .
\end{array}
$$

In the above equation, $\boldsymbol{E}_{e}=\frac{1}{2}\left(\boldsymbol{F}_{e}{ }^{T} \boldsymbol{F}_{e}-\boldsymbol{I}\right)$ is the elastic Green strain tensor, $J^{e}=\operatorname{det} \boldsymbol{F}_{e}$ is the Jacobian of the elastic deformation gradient tensor and $E_{i j}$ with $(i, j) \in(f, s, n)$ are the components of $\boldsymbol{E}_{e}$ that correspond to the material coordinates in the fiber $\boldsymbol{f}$, sheet $\boldsymbol{s}$ and sheet-normal $\boldsymbol{n}$ directions. The material parameters are $C_{\text {compr }}, C, b_{f}$, $b_{s}$ and $b_{f s}$. The passive stress is related to the strain energy function $W$ in (6) by the standard push-forward operation i.e.,

$\boldsymbol{\sigma}_{p}=\frac{1}{J^{e}} \boldsymbol{F}_{e} \cdot \frac{\partial W\left(\boldsymbol{E}_{e}\right)}{\partial \boldsymbol{E}_{e}} \cdot \boldsymbol{F}_{e}^{T}$

\subsection{Integrated electromechanical-reversible growth model}

As discussed in the previous two sections, the reversible growth model was coupled to the electromechanical model via the elastic deformation tensor defined in Eq. (1) and the growth constitutive relationship defined in Eq. (2)-(4). These two equations account for the bidirectional relationship 
between the tissue's local growth and its local mechanical behavior. Herein, we discuss other implementation details associated with the integrated electromechanical-reversible growth model.

Separation of timescale between growth and elastic deformation

The fact that appreciable G\&R can only be observed after a large number of heart beats suggests a separation of timescale between the the reversible growth model (Section 2.1) and the electromechanical model (Section 2.2). Consequently, the growth tensor $\boldsymbol{F}_{g}$ can be treated as a constant within the electromechanical model. Correspondingly, we define a growth cycle as the cumulation of multiple number heart beats within which G\&R is not detectable and does not affect the electromechanics of the heart. By assuming that the heart beats are identical to each other within each growth cycle, the simulation of a cardiac cycle is equivalent to that of a growth cycle.

By this construct, we are able to prescribe mechanical states of the entire cardiac cycle as stimuli in the G\&R model instead of restricting the stimuli to the mechanical state at a distinct point of the cardiac cycle, such as end-diastole in some models (Göktepe et al. 2010a; Lee et al. 2015). This approach is consistent with the modeling philosophy of Kerckhoffs et al. (2012b) where they asserted that individual myocytes should, in principle, be imperceptible to the specific cardiac phase they are in. This approach is also consistent with experiments in which time-varying mechanical stress and strain signals (such as their maximum, mean and amplitude) were found to be likely mechanical candidates that stimulate volume overload G\&R rather than the signals derived at a particular time point in a cardiac cycle (Holmes 2004). Guided by the above-described modeling philosophy and experimental observations, we prescribed the deviation of the time-average elastic myofiber stretch over one growth cycle $\bar{\lambda}_{e}$ from its corresponding homeostatic set point value $\bar{\lambda}_{h}$ (i.e., $\bar{\lambda}_{e}-\bar{\lambda}_{h}$ ) as the driving stimulant of G\&R in our model. As a result, the local value of $\theta$ in each growth cycle can be updated using its previous value via an explicit time integration of Eq. (3) i.e.,

$\theta_{n+1}=\theta_{n}+k\left(\theta_{n}, \bar{\lambda}_{e, n}\right)\left(\bar{\lambda}_{e, n}-\bar{\lambda}_{h}\right)$,

where the subscripts $n$ and $n+1$ denote the growth cycle number.

\section{Elasticity tensors}

Because the growth tensor $\boldsymbol{F}_{g}$ is treated as a constant within each growth cycle (see above Sec- tion), the calculation of the elasticity tensors becomes simplified as a result. By defining the components in the 2nd Piola-Kirchhoff elastic stress tensor as $\partial S_{m n}^{e}=\partial W / \partial E_{m n}^{e}$, the second elasticity tensor $\mathcal{C}_{i j k l}$ in the reference configuration was transformed by a simple pull-back of the elastic moduli $\mathcal{C}_{m n p q}^{e}=\partial S_{m n}^{e} / \partial E_{p q}^{e}$ that is given as follows:

$\mathcal{C}_{i j k l}=\mathcal{C}_{m n p q}^{e} F_{g, i m}^{-1} F_{g, j n}^{-1} F_{g, k p}^{-1} F_{g, l q}^{-1}$.

We note that the above elastic moduli tensor differs from that given in Goktepe et al., (Göktepe et al. 2010a). This is because in their implementation, their growth tensor $\boldsymbol{F}_{g}$ depends on the instantaneous myofiber stretch $\lambda_{e}$ whereas in our implementation, $\boldsymbol{F}_{g}$ is a constant within the growth cycle and does not depend on $\lambda_{e}$.

Similarly, the first elasticity tensor $\mathcal{A}_{i j k l}$ can be transformed by a simple pull-back of the first elastic moduli $\mathcal{A}_{m n p q}^{e}=\partial P_{m n}^{e} / \partial F_{p q}^{e}$ given by

$\mathcal{A}_{i j k l}=\mathcal{A}_{i n k q}^{e} F_{g, j n}^{-1} F_{g, l q}^{-1}$.

This transformation is necessary for integrating the growth model with the electromechanical model because the constitutive law for the active stress is usually defined via the first PiolaKirchhoff or Cauchy stress tensor. As discussed by Sundnes et al. (2014), it is significantly easier to linearize the active stress tensor $\boldsymbol{\sigma}_{a}$ using the first elasticity tensor.

\section{Relieving the residual stresses}

In the volumetric growth framework, the growth tensor $\boldsymbol{F}_{g}$ generates incompatibility that is restored by the elastic deformation gradient tensor $\boldsymbol{F}_{e}$ in the unloaded but not necessary stressfree configuration. Correspondingly, tissue growth induces residual stresses and strains under this framework (Rodriguez et al. 1994; Genet et al. 2015; Lee et al. 2015). The experimental study by Omens et al. (1996), however, showed that the time course of opening angle in the rat's heart did not follow the changes in the thickening of cells during hypertrophy. Their finding, therefore, calls into question of whether tissue growth can by itself alter the residual stress field in the heart. Given that it is still not entirely clear as to whether G\&R can alter the residual stress field in the heart, we have chosen here to relieve the residual stresses that were generated by the incompatible growth tensor $\boldsymbol{F}_{g}$ at each growth cycle.

The residual stresses were relieved by updating the reference (stress-free) nodal position to its new position in the unloaded configuration at the 


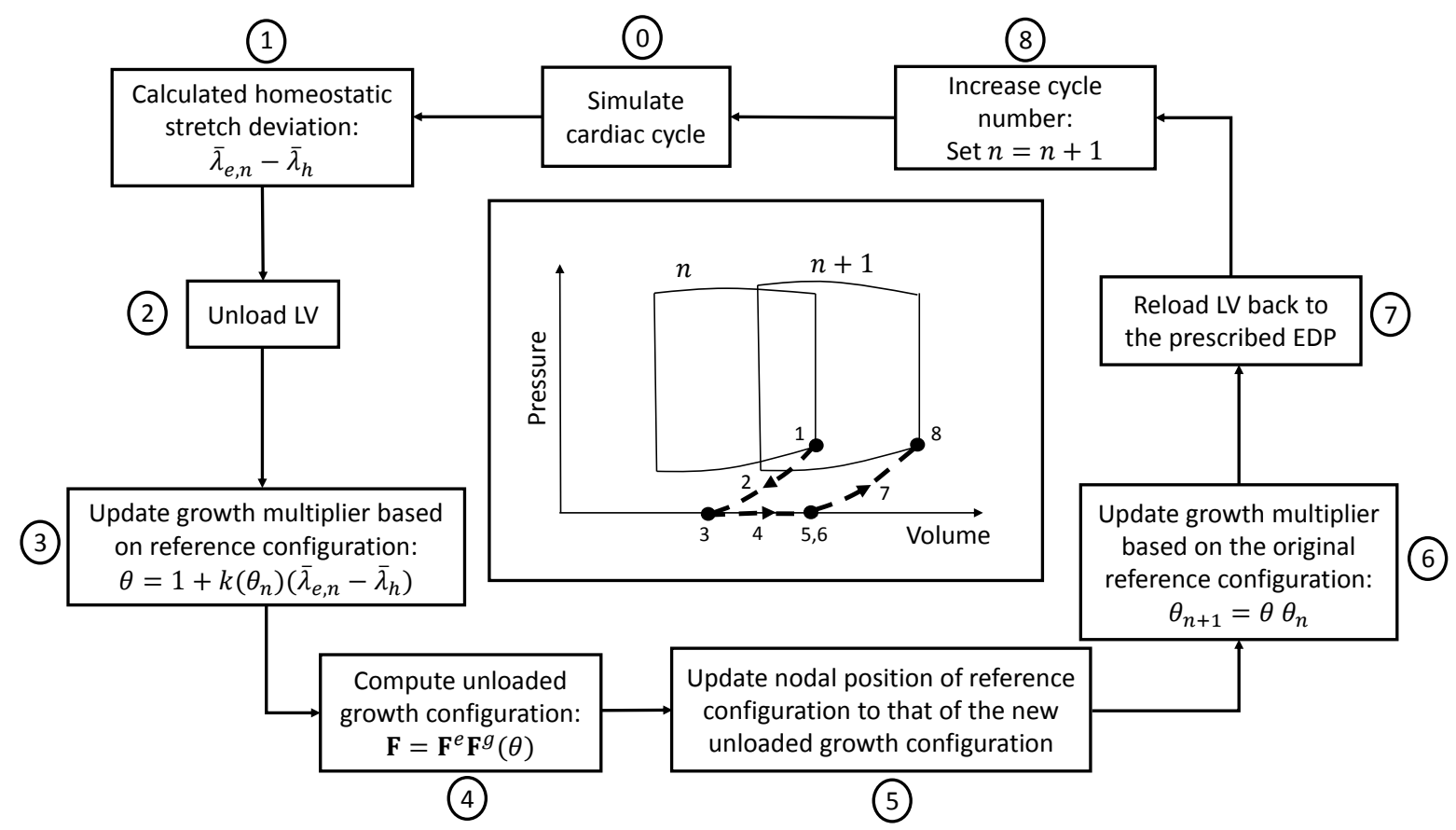

Fig. 1: Scheme to update the growth multiplier $\theta$. Inset shows a sketch of the evolution of PV loop under this scheme.

end of each growth cycle (Steps 3-6 in Figure 1). This method is equivalent to the "updated reference configuration approach" described in Kroon et al. (2009). With this approach, the growth tensor $\boldsymbol{F}_{g}$ is referred to the most recent updated configuration and correspondingly, its input parameter $\theta$ was computed based on this updated configuration (Step 3 of Figure 1). Accordingly, we also note the cumulative growth of all previous cycles $\theta_{n}$ (computed in Step 6 of Figure 1) was used as input in the function $k$ in this step.

2.4 Simulating the long term response of infarct stiffening therapy

We applied the integrated electromechanicalreversible growth model to a realistic human LV geometry to simulate the chronic long term effects of a novel therapy that seeks to attenuate LV remodeling by stiffening the infarct (Morita et al. 2011; Fomovsky et al. 2011). Standard displacement-based finite element (FE) method with an operator splitting scheme was used to solve the system of partial differential equations describing the integrated electromechanicalreversible growth model. Details of the solution scheme can be found in Sundnes et al. (2014).

\section{Left ventricular computational model}

The LV geometry was reconstructed from magnetic resonance images of a patient and a FE mesh consisting of 4425 tetrahedral elements was used to model the LV. In the FE model, the LV epicardial edge was fixed and the LV base was constrained to move only in the basal plane. Helix angle defining the myofiber direction $\boldsymbol{f}$ was prescribed to vary linearly across the LV wall from $-60^{\circ}$ (endocardium) to $60^{\circ}$ (epicardium) (Streeter et al. 1969) (Figure 2a).

\section{Simulating the full cardiac cycle}

We simulated several full cardiac cycles with each having a cycle length of $900 \mathrm{~ms}$. Specifically, passive filling was simulated by incrementally applying pressure to the LV endocardial surface until the prescribed end-diastolic pressure (EDP) was reached. Thereafter, systole was simulated by stimulating the LV apex and its cavity volume was constrained to remain constant during the isovolumic contraction phase. When the LV pressure (LVP) exceeded the prescribed aortic valve opening pressure $P_{a o}$, the LV cavity volume was coupled to a 3 parameter Windkessel model to simulate the ejection phase (Wall et al. 2012) (Figure 2b). The parameters associated with the Windkessel model are the peripheral pressure $P_{p}$, aortic compliance $C_{a o}$, aortic resistance $R_{a o}$ and the peripheral resistance $R_{p}$. Ejection phase was terminated when the LV cavity outflow rate became negative. The same volume constraint was then applied to describe the isovolumic relaxation phase. 

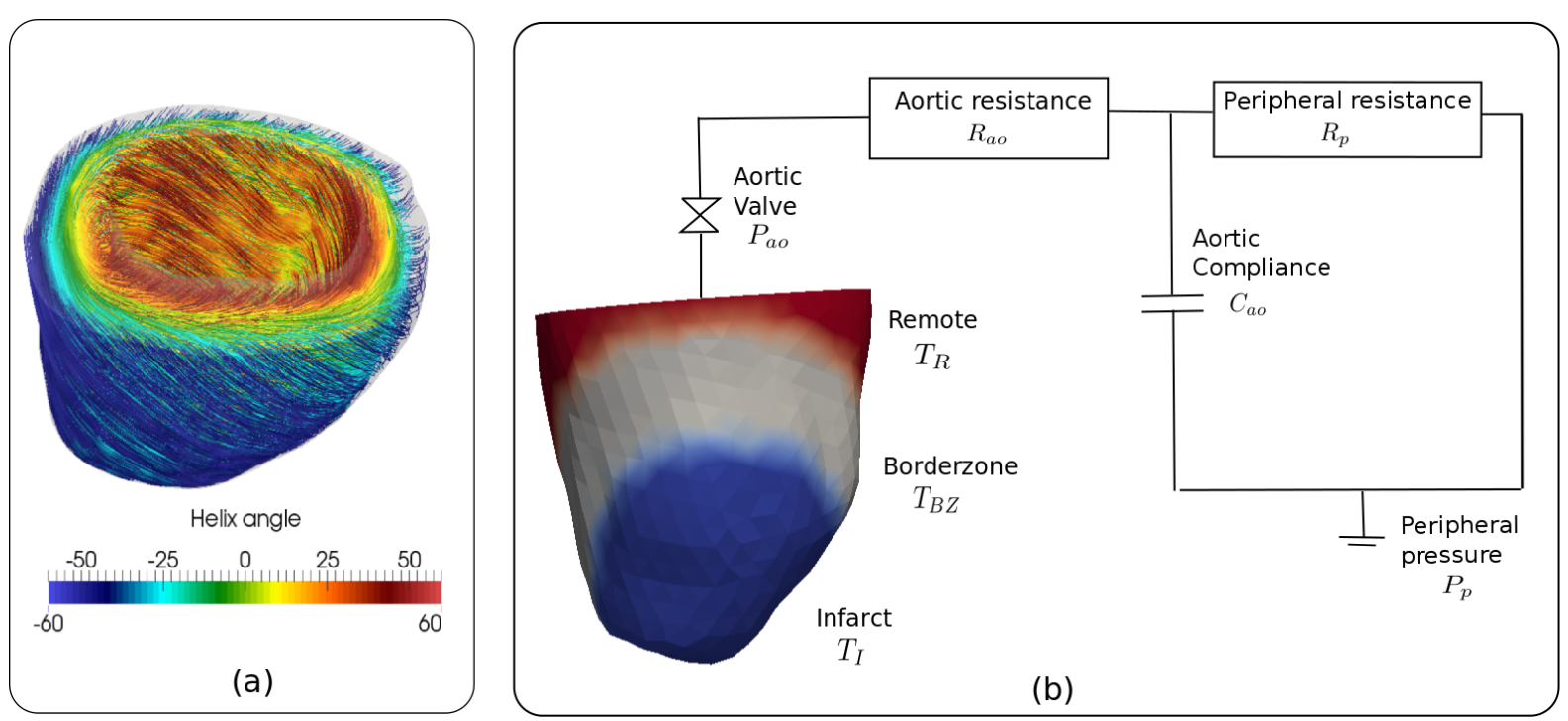

Fig. 2: LV FE model: (a) myofiber direction color coded with the the local helix angle (b) infarcted LV model connected to a 3-parameter Windkessel model to simulate cardiac cycle.

\section{Simulation cases}

Four cases were simulated using the LV FE model. These cases differ from each other in terms of the prescribed values for the $\mathrm{LV}$ passive stiffness $C$ and myocardial contractility that is given by the reference tension $T$ in Eq. (5). The differences are summarized below:

Normal: $\quad$ LV has homogeneous and normal passive stiffness $C_{N}$ and contractility $T_{N}$. (Refer to Table 1 for these values)

I-1 : $\quad$ LV has 3 distinct material regions consisting of an infarct, a borderzone (BZ) and a remote (healthy) region (Figure $\mathbf{2 b}$ ). These regions have similar passive stiffness $C_{N}$ but different contractility $T$. The contractility values at the infarct, BZ and remote were $T_{R}=T_{N}, T_{B Z}=$ $0.5 T_{N}$ and $T_{I}=0 \mathrm{kPa}$, respectively.

$I-10$ : $\quad$ Same as $I-1$ except that $C$ is 10 times higher at the infarct.

$I-100$ : $\quad$ Same as $I-1$ except that $C$ is 100 times higher at the infarct.

All other parameters associated with the simulation are given in Table $\mathbf{1}$. We note that the infarct was assumed to be completely devoid of myocytes and hence, was prohibited from growing (and shrinking) via cellular hypertrophy (and atrophy) in the simulation (i.e., $\theta=1$ ). The infarct LV's regional contractility was prescribed based on a previous study showing that the developed con- tractile force at the $\mathrm{BZ}$ is one-half of that at the remote region (Guccione et al. 2001). We also note that the homeostatic set point for the average elastic myofiber stretch $\bar{\lambda}_{h}$ in the growth constitutive relationship in Eq. (8) was prescribed using the local values obtained from the "Normal" case.

\begin{tabular}{|c|l|}
\hline Circulation & Passive mechanics \\
\hline \hline$R_{a}=15 \mathrm{mmHg} \mathrm{s} / \mathrm{ml}$ & $C_{N}=0.189 \mathrm{kPa}$ \\
$R_{p}=4.5 \mathrm{mmHg} \mathrm{s} / \mathrm{ml}$ & $b_{f f}=29.9$ \\
$C_{a o}=0.4 \mathrm{ml} / \mathrm{mmHg}$ & $b_{s}=13.5$ \\
$P_{p}=4.5 \mathrm{mmHg}$ & $b_{f s}=13.3$ \\
$\mathrm{EDP}=13.5 \mathrm{mmHg}$ & $C_{\mathrm{compr}}=20 \mathrm{kPa}$ \\
$P_{a o}=52.5 \mathrm{mmHg}$ & \\
\hline Growth & Active mechanics \\
\hline \hline$\lambda_{h}=$ "Normal" case & $T_{N}=60 \mathrm{kPa}$ \\
$\gamma_{g}=1$ & *All other parameters \\
$\gamma_{r g}=1$ & are given in Rice et al. \\
$\tau_{g}=0.2$ & (2008) \\
$\tau_{r g}=0.2$ & \\
\hline
\end{tabular}

Table 1: Values of the simulation parameters

\section{Results}

\subsection{Pressure-volume relationship}

Compared to the Normal case, the presence of a non-contractile infarct and a BZ with depressed contractility in the other 3 cases $(I-1, I-10$ and $I-$ 100 ) caused an immediate reduction in pump function (Figure 3). In these cases, the stroke volume (SV) was about half of that of the Normal case.

Besides causing a substantial reduction in the pump function, impairment of the contractility of the infarcted LVs also has an effect on the long term response (Figure 3). The long term response was indicated by a rightward shift of the pressure 

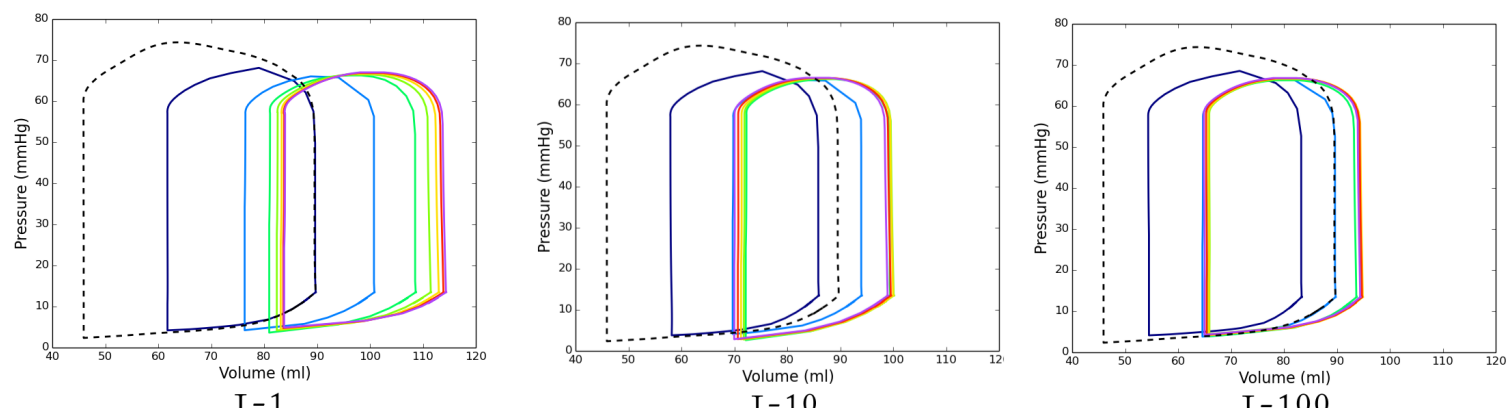

Fig. 3: Effects on the pressure-volume loop for $I-1, I-10$ and $I-100$. Dotted line shows the Normal case.

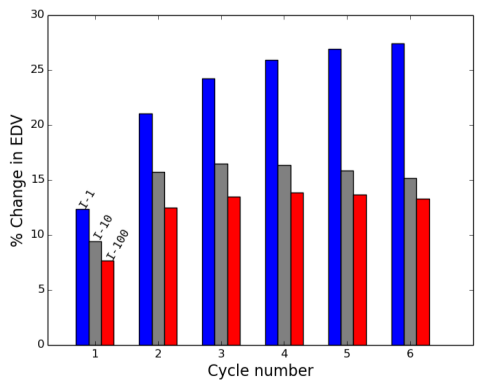

(a)

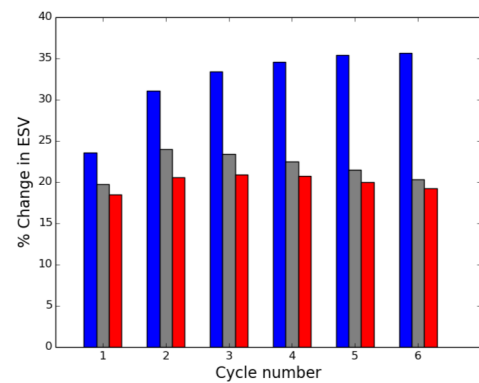

(b)

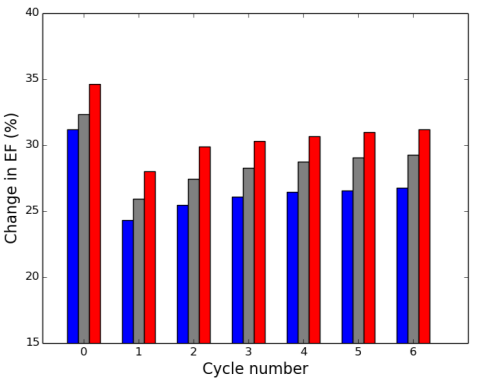

(c)

Fig. 4: Long term effects on (a) end-diastolic volume (a) end-systolic volume and (c) ejection fraction in the $I-1$, $I-10$ and $I-100$ cases.

volume (PV) loop in each growth cycle. The largest shift of the PV loop occurred in the unstiffened infarct case $(I-1)$ in which both end-diastolic volume (EDV) and end-systolic volume (ESV) were increased by about $23 \mathrm{ml}$ at the final growth cycle. The magnitude of the shift decreased with increasing infarct stiffness, and the least amount of shifting of the PV loop occurred in the case with the stiffest infarct $(I-100)$. In that case, both EDV and ESV increased by approximately $10 \mathrm{ml}$.

In terms of the percentage change in EDV and $\mathrm{ESV}$ at the final growth cycle with respect to those at the initial cycle, the $I-1$ case had the largest increase (EDV: $27 \%$ and ESV: $36 \%$ ). On the other hand, the $I-100$ case showed the smallest percentage increase (EDV: $13 \%$ and ESV: 19\%) (Figure 4). Ejection fraction (EF) decreased in the infarct LV cases with $I-1$ and $I-100$ having the largest and smallest decrease of $5 \%$ and $3 \%$, respectively.

\subsection{Geometrical changes}

Corresponding to the rightward shift of the PV loop, the infarcted LV became dilated and more spherical as the ratio of the short-axis to long-axis dimension increased in each growth cycle (Figure 5). Myocytes hypertrophy occurred primarily at the BZ as reflected by the higher value of the growth multiplier $\theta$ found in that region (Figure
6). Consistent with the result that the change in EDV and ESV decreased with increasing infarct stiffness (see previous section), we find that $\theta$ was higher in the $I-1$ case than the $I-10$ and $I-100$ cases (Figure 6a). The higher $\theta$-value in the $I-1$ case with an unstiffened infarct was reflected by the larger and more spherical LV found in the final growth cycle when compared to that in the $I_{-}$ 100 case with a stiffer infarct (Figure 6b). Defining the sphericity $(\mathrm{SI})$ as the ratio between the largest dimension in the long and short axis views,

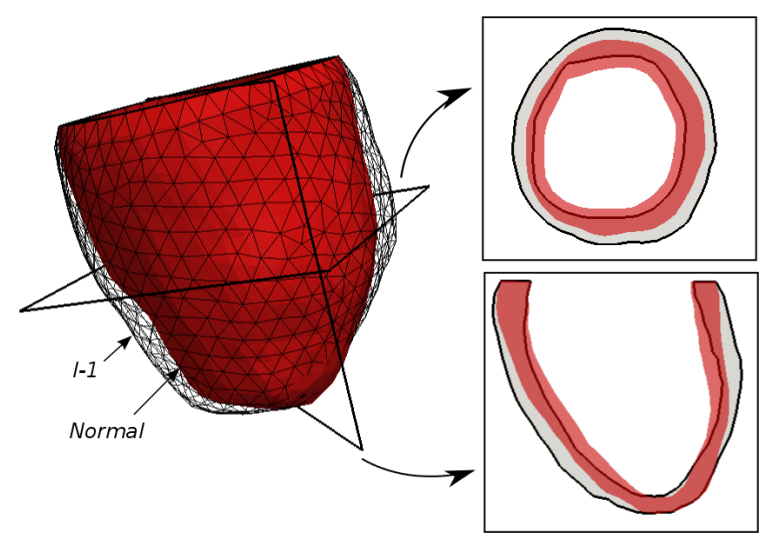

Fig. 5: Comparison of the end-diastolic LV geometry of the final growth cycle between the $I-1$ and Normal cases. Short and long-axis view are shown as insets. Black and red denote $I-1$ and Normal, respectively. 

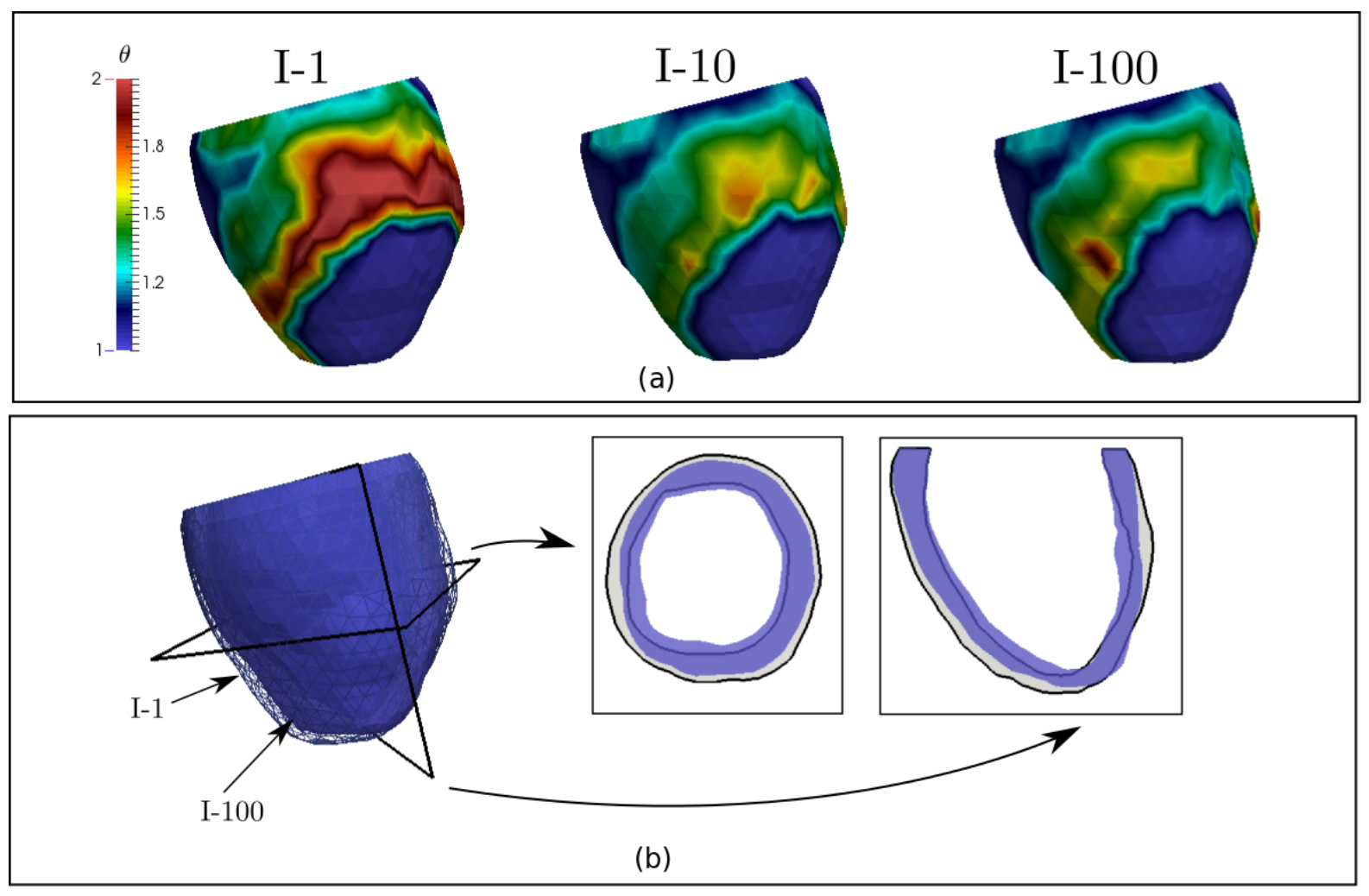

Fig. 6: Comparison of (a): growth multiplier $\theta$ (final cycle) between the cases $I-1, I-10$ and $I-100$. (b): LV geometry at $\mathrm{ED}$ of the final growth cycle between $I-1$ (black) and $I-100$ (blue). Short and long-axis view are shown as insets

sphericity increased by $7.2 \%$ (SI: $0.97 \rightarrow 1.04$ ) in the $I-1$ case and only increased by less than $1 \%$ (SI: $1.01 \rightarrow 1.02)$ in the $I-100$ case. We also note that $\theta$ was unity in the infarct because this region was not permitted to grow in our simulation.

\subsection{Elastic myofiber stretch}

Because the infarct is non-contractile, the elastic myofiber stretch in the infarct region was out-ofphase with the other two regions during the cardiac cycles (Figure 7). When averaged over time in each growth cycle, the elastic myofiber stretch $\bar{\lambda}_{e}$ in the BZ of the infarct $\mathrm{LV}$ cases $(I-1, I-10$ and $I-100)$ was substantially higher when compared to that found in the Normal case. In subsequent cycles involving G\&R, $\bar{\lambda}_{e}$ at the $\mathrm{BZ}$ decreased and became close to the value found in the Normal case (i.e., $\bar{\lambda}_{e, h}$ ). This feature was independent of the infarct stiffness and was found in all the infarct LV cases.

On the other hand, the difference between $\bar{\lambda}_{e}$ in the non-contractile infarct and that found in the Normal case was sensitive to the infarct stiffness. This difference decreased with increasing infarct stiffness. In the $I-1$ case, $\bar{\lambda}_{e}$ was significantly higher in the infarct before the onset of G\&R when compared to that found in the other regions (i.e., $\mathrm{BZ}$ and remote). The time-averaged elastic myofiber stretch $\bar{\lambda}_{e}$ found at the infarct was also higher than that found in the Normal case. This result reflects the effects of having an unstiffened infarct which, without the ability to contract, underwent stretching in the myofiber direction in most part of the cardiac cycle. Because the infarct was prescribed not to grow, the region's time-averaged elastic myofiber stretch $\bar{\lambda}_{e}$ remained elevated in the subsequent growth cycles. With increasing infarct stiffness, however, elastic stretching of the infarct was reduced as found in the $I-10$ and $I-100$ cases. In the $I-100$ case, $\bar{\lambda}_{e}$ at the infarct was comparable to that found the $\mathrm{BZ}$ and remote regions. This value was also close to that found in the Normal case.

\section{Discussion}

We have described the integration of a reversible cardiac growth constitutive model with a coupled electromechanical model of the heart (i.e. integrated electromechanical-reversible growth model). We have also applied this integrated model to simulate the long term effects resulting from an alteration of the infarct mechanical stiffness as a form of therapy. By invoking a separation of timescale between the immediate deformation and the long term G\&R process, we were able to use the deviation of the time-averaged 


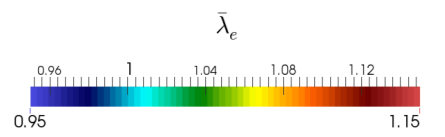

I - 1

$\mathrm{I}-10$

$I-100$
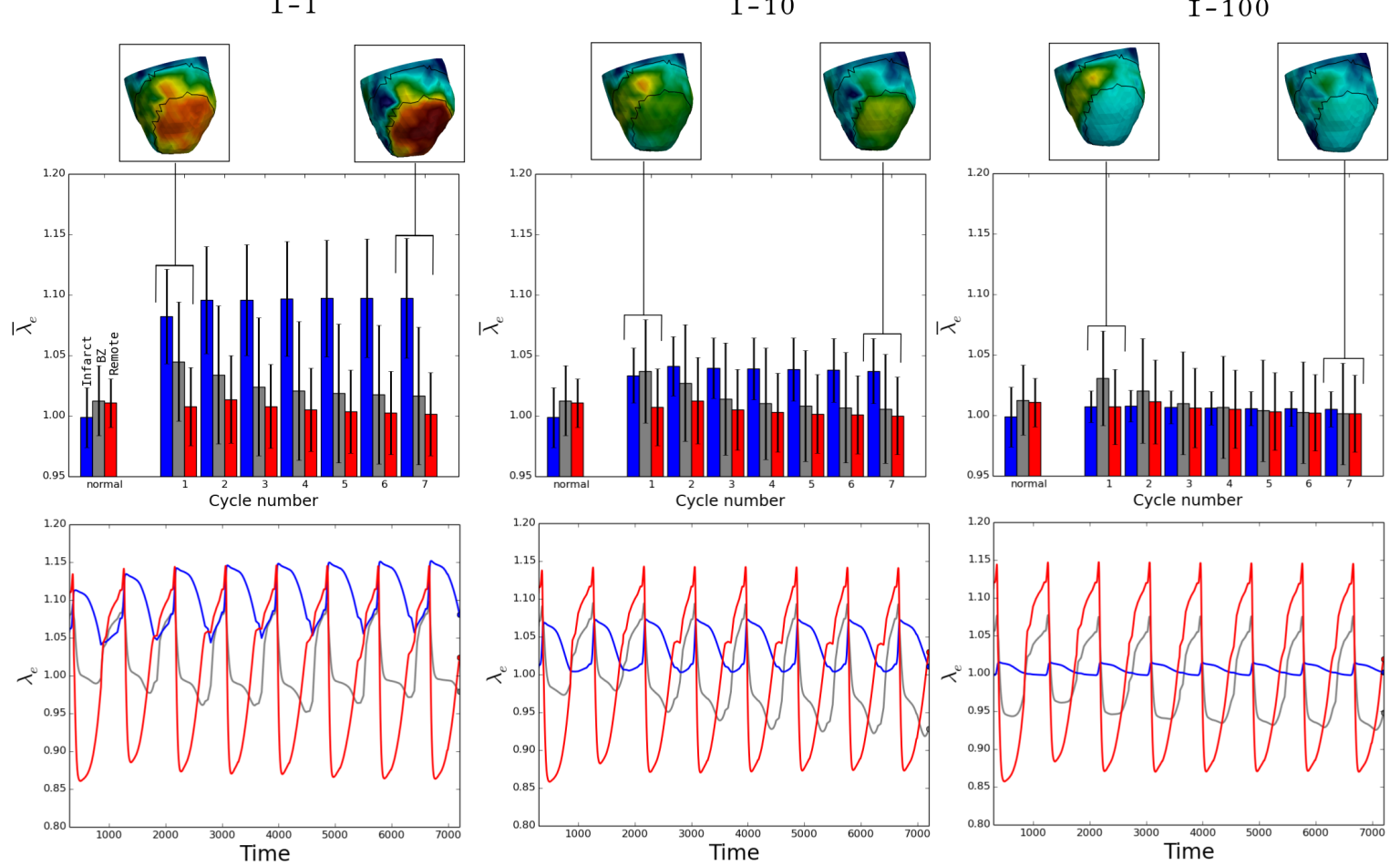

Fig. 7: Long term effects on myofiber stretch in the $I-1, I-10$ and $I-100$ cases. Upper: time-averaged elastic myofiber stretch $\bar{\lambda}_{e}$ of each growth cycle. Lower: local elastic myofiber stretch $\lambda_{e}$ (taken from arbitary points in each material regions) as a function of time over all growth cycles. Blue: infarct, grey: BZ and red: remote.

elastic myofiber strain from its homeostatic set point value as the stimulant for G\&R and update the growth multiplier $\theta$ in each growth cycle. This assumption is appropriate because the timescale at which post MI remodeling occurs (in the order of days to months (Sutton and Sharpe 2000)) is slower by several orders of magnitude than the time taken for a cardiac cycle to complete (in the order of seconds). Moreover, the assumption of timescale separation also simplifies the calculation of the elasticity tensors. The results from our simulation are consistent with experimental and clinical observations of MI in both animals and patients.

\subsection{Effects of MI on local deformation}

First, our simulation shows that in the presence of a non-contractile infarct, the time-averaged elastic myofiber stretch is higher in the adjacent BZ region compared to the homeostatic set point value found in the same region when the LV was normal (Figure 7). Consistent with this result, abnormal stretching of the tissue and reduced negative (contracting) strain magnitude were observed in the
BZ in animal models and patients during systole (Kramer et al. 1993; Ashikaga et al. 2005; Rutz et al. 2008; Soleimanifard et al. 2012). When averaged over a cardiac cycle, the BZ elastic myofiber stretch would, therefore, be higher than that found in the remote region.

\subsection{Effects of MI on local G\&R}

Second, the elevated elastic myofiber stretch found in the $\mathrm{BZ}$ region drives the local G\&R process that occurs predominantly in this region itself, as reflected by its higher $\theta$ value (Figure 6). Because the field variable $\theta$ reflects local growth in the myofiber direction, this result implies that the myocytes became larger and elongated in the borderzone region. Likewise in both MI animal models (Jackson et al. 2002; Shimkunas et al. 2014) and patients (Gerdes et al. 1992), surviving myocytes in the infarcted LV typically undergo hypertrophy and became larger. In a histological study of the infarcted sheep heart, the myocytes was found to be about $70 \%$ larger than that in the remote region 2 weeks after MI (Shimkunas et al. 2014). Similarly, myocytes isolated from explanted hearts of 
patients who had ischemic dilated cardiomyopathy were observed to be about $40 \%$ longer than those found in the normal human heart (Gerdes et al. 1992).

\subsection{Effects of MI on global G\&R}

Third, our simulation shows that the collective effects of local tissue growth in the non-infarcted region led to the LV becoming dilated and markedly more spherical (Figure 5), a feature that corresponds to a rightward shift of the LV pressure volume loop in each growth cycle (Figure 3). This pathological feature of progressive LV dilation due to the presence of an MI has been observed. Specifically, dilation of LV was found in MI patients and animal models to be accompanied by a rightward shift of the pressure-volume loop and a parallel increase in both EDV and ESV (Warren et al. 1988; Jeremy et al. 1989; Sutton and Sharpe 2000; McCall et al. 2012).

\subsection{Effects of altering the mechanical properties} of MI

Last, our simulation shows that the remodeling rate, defined by the rate of increase in EDV and ESV, decreases with increasing mechanical stiffness of the infarct. In other words, our simulation predicts that LV remodeling can be attenuated by stiffening the infarct (Figure 4). As suggested by our simulation, a possible mechanism for this effect is that the stiffened infarct reduces the time-average elastic myofiber stretch in the BZ region (Figure $\mathbf{7}$ ). This prediction is consistent with a number of long-term large animal studies that were conducted by different groups to investigate the alteration of infarct mechanical properties as a treatment for MI (Mukherjee et al. 2008; Ifkovits et al. 2010; Morita et al. 2011). Different materials were injected directly to stiffen the infarct in these studies. These materials include fibrin-alginate (Mukherjee et al. 2008), methacrylated hyaluronic acid macromers (MeHA) (Ifkovits et al. 2010) and a biocompatible dermal filler of calcium hydroxyapatite (Morita et al. 2011). Results from these experimental studies show that post-MI remodeling was attenuated when the infarct was stiffened. In particular, a smaller increase in EDV and ESV was found in treated animals when compared to the untreated animals after several weeks. In Morita et al. (2011), LV remodeling was found to be attenuated at 8-week follow up in animals that underwent this therapy when compared to those that did not. In that study, the increase in LV volumes was found to be lower in treated animals (ESV : $39 \%$ and EDV : $28 \%$ ) compared to untreated animals (ESV : $83 \%$ and EDV : $65 \%$ ). Cardiac function, as quantified by the ejection fraction (EF), was also found to have deterioriated at a slower rate in treated animals (EF: $36 \% \rightarrow 31 \%$ ) compared to untreated animals (EF: $35 \% \rightarrow 28 \%$ ). The stiffness of the infarct was also found to be an important parameter that affects the outcome of this therapy in the experimental study by Ifkovits et al. (Ifkovits et al. 2010). In their study, which consists of two groups of animals injected with different amounts of MeHA, they found that cardiac function and remodeling rate was significantly improved only in animals injected with a higher amount of MeHA that correspond to a stiffer infarct.

\subsection{Conclusion}

In summary, we have presented an integrated electromechanical-reversible growth model and have applied it to study the long term effects resulting from altering the infarct mechanical properties. This work overcomes the limitation of a previous study by Fomovsky et al. (2011), in which only the immediate (acute) effects of this therapy was modeled. We have demonstrated that the simulation results obtained from the integrated model are qualitatively consistent with the key experimental and clinical findings of both MI-induced remodeling and infarct stiffening therapy. Future studies will investigate the effects of altering the mechanical anisotropy of the infarct as an experimental study using animal model have shown that the acute pump function is sensitive to this property (Fomovsky et al. 2012).

\section{Acknowledgement}

This work was supported by the Marie Curie International Outgoing Fellowship within the 7th European Community Framework Program (M. Genet).

\section{References}

Ashikaga, H., Mickelsen, S. R., Ennis, D. B., Rodriguez, I., Kellman, P., Wen, H. and McVeigh, E. R. (2005), "Electromechanical analysis of infarct border zone in chronic myocardial infarction.", Am. J. Physiol. Heart Circ. Physiol. 289, H1099-H1105.

Athanasuleas, C. L., Buckberg, G. D., Stanley, A. W. H., Siler, W., Dor, V., Di Donato, M., Menicanti, L., Almeida de Oliveira, S., Beyersdorf, F., Kron, I. L., Suma, H., Kouchoukos, N. T., Moore, 
W., McCarthy, P. M., Oz, M. C., Fontan, F., Scott, M. L. and Accola, K. a. (2004), "Surgical ventricular restoration in the treatment of congestive heart failure due to post-infarction ventricular dilation.", J. Am. Coll. Cardiol. 44(7), 1439-45.

Cohn, J. N., Ferrari, R. and Sharpe, N. (2000), "Cardiac remodeling concepts and clinical implications: a consensus paper from an international forum on cardiac remodeling", J. Am. Coll. Cardiol. 35(3), 569-582.

Fomovsky, G. M., Clark, S. A., Parker, K. M., Ailawadi, G. and Holmes, J. W. (2012), "Anisotropic reinforcement of acute anteroapical infarcts improves pump function.", Circ. Heart Fail. 5(4), 515-22.

Fomovsky, G. M., MacAdangdang, J. R., Ailawadi, G. and Holmes, J. W. (2011), "Model-based design of mechanical therapies for myocardial infarction", $J$. Cardiovasc. Transl. Res. 4, 82-91.

Genet, M., Lee, L. C., Nguyen, R., Haraldsson, H., Acevedo-Bolton, G., Zhang, Z., Ge, L., Ordovas, K., Kozerke, S. and Guccione, J. M. (2014), "Distribution of normal human left ventricular myofiber stress at end diastole and end systole: a target for in silico design of heart failure treatments.", J. Appl. Physiol. 117, 142-52.

Genet, M., Rausch, M. K., Lee, L. C., Choy, J. S., Zhao, X., Kassab, G., Kozerke, S., Guccione, J. and Kuhl, E. (2015), "Heterogeneous growth-induced prestrain in the heart", J. Biomech. (In press).

Gerdes, A. M., Kellerman, S. E., Moore, J. A., Muffly, K. E., Clark, L. C., Reaves, P. Y., Malec, K. B., McKeown, P. P. and Schocken, D. D. (1992), "Structural remodeling of cardiac myocytes in patients with ischemic cardiomyopathy", Circulation 86(2), 426-430.

Göktepe, S., Abilez, O. J. and Kuhl, E. (2010b), "A generic approach towards finite growth with examples of athlete's heart, cardiac dilation, and cardiac wall thickening", J. Mech. Phys. Solids 58(10), 16611680 .

Göktepe, S., Abilez, O. J., Parker, K. K. and Kuhl, E. (2010a), "A multiscale model for eccentric and concentric cardiac growth through sarcomerogenesis.", J. Theor. Biol. 265(3), 433-42.

Guccione, J. M., McCulloch, A. D. and Waldman, L. K. (1991), "Passive material properties of intact ventricular myocardium determined from a cylindrical model.", J. Biomech. Eng. 113(1), 42-55.

Guccione, J. M., Moonly, S. M., Moustakidis, P., Costa, K. D., Moulton, M. J., Ratcliffe, M. B. and Pasque, M. K. (2001), "Mechanism underlying mechanical dysfunction in the border zone of left ventricular aneurysm: a finite element model study.", Ann. Thorac. Surg. 71(2), 654-62.

Heidenreich, P. A., Trogdon, J. G., Khavjou, O. A., Butler, J., Dracup, K., Ezekowitz, M. D., Finkelstein, E. A., Hong, Y., Johnston, S. C., Khera, A., LloydJones, D. M., Nelson, S. A., Nichol, G., Orenstein, D., Wilson, P. W. F. and Woo, Y. J. (2011), "Forecasting the future of cardiovascular disease in the United States: A policy statement from the American Heart Association", Circulation 123, 933-944.

Holmes, J. W. (2004), "Candidate mechanical stimuli for hypertrophy during volume overload.", J. Appl. Physiol. 97(May 2004), 1453-1460.

Hoshikawa, E., Matsumura, Y., Kubo, T., Okawa, M., Yamasaki, N., Kitaoka, H., Furuno, T., Takata, J. and Doi, Y. L. (2011), "Effect of left ventricular reverse remodeling on long-term prognosis after therapy with angiotensin-converting enzyme inhibitors or angiotensin II receptor blockers and $\beta$ blockers in patients with idiopathic dilated cardiomyopathy.", Am. J. Cardiol. 107(7), 1065-1070.

$\mathrm{Hu}$, Y., Gurev, V., Constantino, J. and Trayanova, N. (2014), "Optimizing cardiac resynchronization therapy to minimize ATP consumption heterogeneity throughout the left ventricle: A simulation analysis using a canine heart failure model", Hear. Rhythm 11, 1063-1069.

Ifkovits, J. L., Tous, E., Minakawa, M., Morita, M., Robb, J. D., Koomalsingh, K. J., Gorman, J. H., Gorman, R. C. and Burdick, J. A. (2010), "Injectable hydrogel properties influence infarct expansion and extent of postinfarction left ventricular remodeling in an ovine model.", Proc. Natl. Acad. Sci. U. S. A. 107, 11507-11512.

Jackson, B. M., Gorman, J. H., Moainie, S. L., Guy, T. S., Narula, N., Narula, J., John-Sutton, M. G., Edmunds, L. H. and Gorman, R. C. (2002), "Extension of borderzone myocardium in postinfarction dilated cardiomyopathy.", J. Am. Coll. Cardiol. 40(6), 1160-7; discussion 1168-71.

Jeremy, R. W., Allman, K. C., Bautovitch, G. and Harris, P. J. (1989), "Patterns of left ventricular dilation during the six months after myocardial infarction.", J. Am. Coll. Cardiol. 13(2), 304-310.

Kerckhoffs, R. C. P., Lumens, J., Vernooy, K., Omens, J. H., Mulligan, L. J., Delhaas, T., Arts, T., McCulloch, A. D. and Prinzen, F. W. (2008), "Cardiac resynchronization: insight from experimental and computational models.", Prog. Biophys. Mol. Biol. 97(2-3), 543-61.

Kerckhoffs, R. C. P., Omens, J. H. and McCulloch, A. D. (2012a), "Mechanical discoordination increases continuously after the onset of left bundle branch block despite constant electrical dyssynchrony in a computational model of cardiac electromechanics and growth", Europace 14.

Kerckhoffs, R. C. P., Omens, J. and McCulloch, A. D. (2012b), "A single strain-based growth law predicts concentric and eccentric cardiac growth during pressure and volume overload.", Mech. Res. Commun. 42, 40-50.

Klepach, D., Lee, L. C., Wenk, J. F., Ratcliffe, M. B., Zohdi, T. I., Navia, J. A., Kassab, G. S., Kuhl, E. and Guccione, J. M. (2012), "Growth and remodeling of the left ventricle: A case study of myocardial infarction and surgical ventricular restoration.", Mech. Res. Commun. 42, 134-141.

Kramer, C. M., Lima, J. A., Reichek, N., Ferrari, V. A., Llaneras, M. R., Palmon, L. C., Yeh, I. T., Tallant, B. and Axel, L. (1993), "Regional differences in function within noninfarcted myocardium during left ventricular remodeling.", Circulation 88, 12791288.

Kroon, W., Delhaas, T., Arts, T. and Bovendeerd, P. (2009), "Computational modeling of volumetric soft tissue growth: application to the cardiac left ventricle.", Biomech. Model. Mechanobiol. 8(4), 301-9.

Lee, L. C., Ge, L., Zhang, Z., Pease, M., Nikolic, S. D., Mishra, R., Ratcliffe, M. B. and Guccione, J. M. (2014a), "Patient-specific finite element modeling of the Cardiokinetix Parachute device: Effects on left ventricular wall stress and function", Med. Biol. Eng. Comput. 52, 557-566.

Lee, L. C., Genet, M., Acevedo-Bolton, G., Ordovas, K., Guccione, J. M. and Kuhl, E. (2015), "A computational model that predicts reverse growth in response to mechanical unloading", Biomech. Model. Mechanobiol. 14(2), 217-229. 
Lee, L. C., Genet, M., Dang, A. B., Ge, L., Guccione, J. M. and Ratcliffe, M. B. (2014c), "Applications of computational modeling in cardiac surgery", $J$. Card. Surg. 29, 293-302.

Lee, L. C., Wall, S. T., Genet, M., Hinson, A. and Guccione, J. M. (2014b), "Bioinjection treatment: Effects of post-injection residual stress on left ventricular wall stress", J. Biomech. 47(12), 3115-3119.

Lee, L. C., Wall, S. T., Klepach, D., Ge, L., Zhang, Z., Lee, R. J., Hinson, A., Gorman, J. H., Gorman, R. C. and Guccione, J. M. (2013b), "Algisyl-LVR with coronary artery bypass grafting reduces left ventricular wall stress and improves function in the failing human heart.", Int. J. Cardiol. 168, 20222028 .

Lee, L. C., Wenk, J. F., Zhong, L., Klepach, D., Zhang, Z., Ge, L., Ratcliffe, M. B., Zohdi, T. I., Hsu, E., Navia, J. L., Kassab, G. S. and Guccione, J. M. (2013a), "Analysis of Patient-specific Surgical Ventricular Restoration - Importance of an Ellipsoidal Left Ventricular Geometry for Diastolic and Systolic Function.", J. Appl. Physiol. (115), 136-144.

McCall, F. C., Telukuntla, K. S., Karantalis, V., Suncion, V. Y., Heldman, A. W., Mushtaq, M., Williams, A. R. and Hare, J. M. (2012), "Myocardial infarction and intramyocardial injection models in swine", Nat. Protoc. 7(8), 1479-1496.

Morita, M., Eckert, C. E., Matsuzaki, K., Noma, M., Ryan, L. P., Burdick, J. A., Jackson, B. M., Gorman, J. H., Sacks, M. S. and Gorman, R. C. (2011), "Modification of infarct material properties limits adverse ventricular remodeling.", Ann. Thorac. Surg. 92(2), 617-24.

Mukherjee, R., Zavadzkas, J. A., Saunders, S. M., McLean, J. E., Jeffords, L. B., Beck, C., Stroud, R. E., Leone, A. M., Koval, C. N., Rivers, W. T., Basu, S., Sheehy, A., Michal, G. and Spinale, F. G. (2008), "Targeted Myocardial Microinjections of a Biocomposite Material Reduces Infarct Expansion in Pigs", Ann. Thorac. Surg. 86, 1268-1276.

Niederer, S. A. and Smith, N. P. (2012), "At the heart of computational modelling.", J. Physiol. 590, 1331-8.

Nikolic, S. D., Khairkhahan, A., Ryu, M., Champsaur, G., Breznock, E. and Dae, M. (2009), "Percutaneous Implantation of an Intraventricular Device for the Treatment of Heart Failure: Experimental Results and Proof of Concept", J. Card. Fail. 15, 790-797.

Omens, J. H., Rodriguez, E. K. and McCulloch, A. D. (1996), "Transmural Changes in Stress-free Myocyte Morphology During Pressure Overload Hypertrophy in the Rat", J. Mol. Cell. Cardiol. 28, 1975-1983.

Rice, J. J., Wang, F., Bers, D. M. and de Tombe, P. P. (2008), "Approximate model of cooperative activation and crossbridge cycling in cardiac muscle using ordinary differential equations.", Biophys. J. 95(5), 2368-90.

Rodriguez, E. K., Hoger, A. and McCulloch, A. D. (1994), "Stress-dependent finite growth in soft elastic tissues", J. Biomech. 27(4), 455-467.

Rutz, A. K., Ryf, S., Plein, S., Boesiger, P. and Kozerke, S. (2008), "Accelerated whole-heart 3D CSPAMM for myocardial motion quantification.", Magn. Reson. Med. 59(4), 755-63.

Sabbah, H., Wang, M., Jiang, A., IIsar, I., Sabbah, M., Helgerson, S., Peterson, R., Tarazona, N. and Lee, R. (2009), "Circumferential mid-ventricular intramyocardial injections of alginate hydrogel improve left ventricular function and prevent progressive remodeling in dogs with chronic heart failure", Circulation 120, S912.
Shimkunas, R., Makwana, O., Spaulding, K., Bazargan, M., Khazalpour, M., Takaba, K., Soleimani, M., Myagmar, B.-E., Lovett, D. H., Simpson, P. C., Ratcliffe, M. B. and Baker, A. J. (2014), "Myofilament dysfunction contributes to impaired myocardial contraction in the infarct border zone", AJP Hear. Circ. Physiol. 307, H1150-H1158.

Soleimanifard, S., Abd-Elmoniem, K. Z., Sasano, T., Agarwal, H. K., Abraham, M. R., Abraham, T. P. and Prince, J. L. (2012), "Three-dimensional regional strain analysis in porcine myocardial infarction: a 3T magnetic resonance tagging study.", $J$. Cardiovasc. Magn. Reson. 14(1), 85.

Streeter, D. D., Spotnitz, H. M., Patel, D. P., Ross, J. and Sonnenblick, E. H. (1969), "Fiber orientation in the canine left ventricle during diastole and systole.", Circ. Res. 24(3), 339-47.

Sundnes, J., Wall, S. T., Osnes, H., Thorvaldsen, T. and McCulloch, A. D. (2014), "Improved discretisation and linearisation of active tension in strongly coupled cardiac electro-mechanics simulations.", Comput. Methods Biomech. Biomed. Engin. 17(6), 604-15.

Sutton, M. and Sharpe, N. (2000), "Clinical Cardiology : New Frontiers Left Ventricular Remodeling After Myocardial Infarction Pathophysiology and Therapy", Circulation 101, 2981-2988.

Wall, S. T., Guccione, J. M., Ratcliffe, M. B. and Sundnes, J. S. (2012), "Electromechanical feedback with reduced cellular connectivity alters electrical activity in an infarct injured left ventricle: a finite element model study.", Am. J. Physiol. Heart Circ. Physiol. 302(1), H206-14.

Wall, S. T., Walker, J. C., Healy, K. E., Ratcliffe, M. B. and Guccione, J. M. (2006), "Theoretical impact of the injection of material into the myocardium: A finite element model simulation", Circulation 114, 2627-2635.

Warren, S. E., Royal, H. D., Markis, J. E., Grossman, W. and McKay, R. G. (1988), "Time course of left ventricular dilation after myocardial infarction: influence of infarct-related artery and success of coronary thrombolysis.", J. Am. Coll. Cardiol. 11(9), 1219.

Wenk, J. F., Wall, S. T., Peterson, R. C., Helgerson, S. L., Sabbah, H. N., Burger, M., Stander, N., Ratcliffe, M. B. and Guccione, J. M. (2009), "A method for automatically optimizing medical devices for treating heart failure: designing polymeric injection patterns.", J. Biomech. Eng. 131(December 2009), 121011.

Williams, A. R., Trachtenberg, B., Velazquez, D. L., McNiece, I., Altman, P., Rouy, D., Mendizabal, A. M., Pattany, P. M., Lopera, G. a., Fishman, J., Zambrano, J. P., Heldman, A. W. and Hare, J. M. (2011), "Intramyocardial stem cell injection in patients with ischemic cardiomyopathy: functional recovery and reverse remodeling.", Circ. Res. 108(7), 792-6.

Winslow, R. L., Rice, J., Jafri, S., Marban, E. and O'Rourke, B. (1999), "Mechanisms of Altered Excitation-Contraction Coupling in Canine Tachycardia-Induced Heart Failure, II : Model Studies", Circ. Res. 84(5), 571-586.

Yu, C.-M., Bleeker, G. B., Fung, J. W.-H., Schalij, M. J., Zhang, Q., van der Wall, E. E., Chan, Y.-S., Kong, S.-L. and Bax, J. J. (2005), "Left ventricular reverse remodeling but not clinical improvement predicts long-term survival after cardiac resynchronization therapy.", Circulation 112, 1580-1586. 